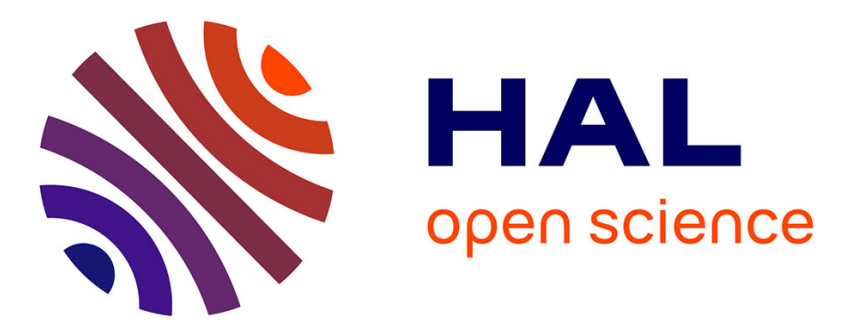

\title{
Investissement narcissique, figure du double et fantasme d'enfant meurtrier
}

\author{
Mireille Guittonneau
}

\section{To cite this version:}

Mireille Guittonneau. Investissement narcissique, figure du double et fantasme d'enfant meurtrier. Cliniques méditerranéennes, 2012, 86, pp.33 - 44. 10.3917/cm.086.0033 . hal-01496816

\section{HAL Id: hal-01496816 https://hal.science/hal-01496816}

Submitted on 27 Mar 2017

HAL is a multi-disciplinary open access archive for the deposit and dissemination of scientific research documents, whether they are published or not. The documents may come from teaching and research institutions in France or abroad, or from public or private research centers.
L'archive ouverte pluridisciplinaire HAL, est destinée au dépôt et à la diffusion de documents scientifiques de niveau recherche, publiés ou non, émanant des établissements d'enseignement et de recherche français ou étrangers, des laboratoires publics ou privés. 


\section{Investissement narcissique, figure du double et fantasme d'enfant meurtrier}

\section{Mireille Guittonneau ${ }^{1}$}

Freud, dans L'inquiétant ${ }^{2}$, présente la figure du double comme étant particulièrement apte à susciter un sentiment d'unheimlich. Il indique, à partir des travaux de Rank, que cette représentation constitue originellement une protection, un démenti de l'état de mortel, avant d'évoluer généralement en signe annonciateur de mort. Qu'en est-il alors de cette figure du double lorsque pour certains sujets, la représentation de la séparation, de la différenciation se confond avec celle d'un danger de mort? Comment prend-elle place dans les relations parents-enfant, en particulier ? La figure du double semble alors constituer pour certains une défense centrale, seule capable d'écarter la dimension traumatique d'une séparationindividuation. Portée par un investissement narcissique quasi exclusif, cette figure du double semble parfois accomplir un meurtre psychique, privant souvent celui qui en est affublé de toute possibilité d'exister. Véritable ombre portée par l'enfant mis en position de double, elle déploie les mailles d'un filet duquel l'enfant risque fort de ne pouvoir se dégager seul. En effet, prisonnier d'un miroir dans lequel son image se trouve sans cesse effacée par celle des autres, les présents, les absents ou les défunts, que lui reste-t-il, sinon à se glisser dans une peau étrangère, une véritable peau de chagrin, rendant impossible toute émergence d'un désir propre, sauf à risquer la mort ?

Nous ferons l'hypothèse, à partir de plusieurs psychothérapies d'enfants, que derrière cet apparent meurtre psychique, peut se dissimuler le fantasme d'un enfant meurtrier ; fantasme dont nous essaierons de montrer les différents ressorts. Nous verrons aussi que ce fantasme prend de multiples déguisements derrière lesquels il est essentiel de le reconnaître afin d'entendre les enjeux vitaux qui se jouent dans ces relations parents-enfant.

\section{L'investissement narcissique dans la relation parents-enfant et ses aléas}

Freud, après avoir reconnu l'existence d'une libido narcissique, a souligné la part du narcissisme qui s'exprime dans toute relation parents-enfant, soulignant que « his majesty the baby», cette part de soi à laquelle chacun est contraint de renoncer, renaît sous l'apparence d'une relation objectale et permet ainsi aux parents de s'accorder une parcelle d'éternité ${ }^{3}$.

\footnotetext{
${ }^{1}$ Psychologue clinicienne, Psychanalyste, Docteur en psychopathologie et psychanalyse, rattachée au CEPP (EA 2374), Université Paris Diderot, Sorbonne Paris Cité.

${ }^{2}$ FREUD, S. 1919. L'inquiétant dans Euvres complètes, t. XV, Paris, PUF, 1996 147-188

${ }^{3}$ FREUD, S. 1914. Pour introduire le narcissisme, dans Euvres complètes, t. XII, Paris, PUF, 2005, 214-245.
} 
Mais après lui, d'autres ont mis l'accent sur les pathologies de l'enfance qui naissent lorsque l'investissement narcissique prédomine, sans laisser de véritable place à un investissement objectal, fondamental pour que se développe la subjectivité de l'enfant. Ainsi Racamier a-t-il fait l'hypothèse que la rencontre de la mère et de son nourrisson s'opère par un mouvement réciproque de séduction narcissique; terme destiné à mettre en exergue le mouvement dynamique qui a lieu. "Pour chacun des partenaires, il s'agit donc d'attirer l'autre à soi et à part. Il s'agit d'établir une relation qui non seulement soit exclusive, mais à l'écart du monde et de son train. ${ }^{4}$ » Fondée sur une symétrie de la relation, la séduction narcissique vise à amoindrir les différences entre mère et enfant et à soutenir leur échange. En cela, elle est tout à fait essentielle aux débuts de la vie du nourrisson. Mais ensuite, il est tout aussi fondamental que ce mouvement décroisse, se retire à l'arrière-plan de la relation, ce qui demande à la mère un travail de maturation : "En sevrant son enfant, elle sèvre aussi celui qu'elle est elle-même par une étroite identification; il lui faut donc renoncer à toute la part narcissique naguère dominante, de sa relation avec l'enfant ${ }^{5}$ ». Elle peut dès lors le considérer "comme un objet distinct et une personne en soi $^{6} »$. L'héritage de cette séduction première se manifeste ensuite dans le sentiment d'être bien dans le monde, dans la capacité à être en empathie, à donner et recevoir de la tendresse. Mais, lorsque ce renoncement s'avère impossible, la séduction narcissique est parfois mise au service du seul narcissisme parental. Toute symétrie disparaît alors et le risque de manipulation de l'objet devient majeur. Au lieu de fonder l'organisation psychique de l'enfant, la séduction narcissique entrave son développement, sa capacité à exister en toute subjectivité. C'est sur un tel fondement que se construisent les fonctionnements incestuels, voire incestueux.

Plus récemment, J. Manzano, F. Palacio Espasa et N. Zilkha ${ }^{7}$ ont montré l'existence de «scénarios narcissiques de la parentalité » et souligné leur influence sur la relation naissante et ses éventuelles distorsions. Certains de ces scénarios comportent un double mouvement : celui de la projection sur l'enfant d'une image du soi (carencé, abandonné, idéalisé ou endommagé) du parent et celui d'une identification de ce même parent à une image complémentaire. Ainsi, à l'image du soi carencé, peut s'associer une image de parent devant éviter toute frustration à son enfant. D'autres scénarios reposent sur la projection, par le

\footnotetext{
${ }^{4}$ RACAMIER, P.C. 1995. L'inceste et l'incestuel, Paris, Les Editions du collège, p. 24.

${ }^{5}$ RACAMIER, P.C. 1979. La maternalité psychotique, dans De psychanalyse en psychiatrie, Paris, Payot, p. 198.

${ }^{6}$ Ibid, p. 198.

${ }^{7}$ MANZANO, J., PALACIO ESPASA, F. et ZILKHA N. 1999. Les scénarios narcissiques de la parentalité, Paris, PUF.
} 
parent, d'un objet interne qui, lui aussi, peut avoir été vécu comme endommagé, idéalisé ou négatif. Mais, selon ces auteurs, le narcissisme parental n'est pas absent de l'objet interne projeté sur l'enfant. En effet, soulignent-ils, les objets internes apparaissent comme une formation composite combinant l'image de leurs propres parents et celle de leur soi infantile. Ces différents scénarios, indiquent les auteurs, ont deux buts : 1) établir une scène et tenter à tout prix de la faire exister et de la rendre immuable (l'image de soi idéal par exemple). 2) Corriger un passé jugé inacceptable. Face à ces pressions fantasmatiques, l'enfant réagit en fonction de ses propres besoins (d'attachement, de holding) liés à ses pulsions et défenses. Il s'identifie alors, totalement ou partiellement, à la représentation projetée sur lui ou rejette le rôle qui lui est attribué et réclame d'être reconnu dans son altérité. C'est alors que des symptômes peuvent surgir et que les parents mènent parfois leur enfant en consultation, car ce refus met en péril l'équilibre parental.

\section{La figure du double comme équivalent d'un meurtre psychique}

On voit comment, au fil de ces quelques travaux, les distorsions entraînées par la prépondérance d'un investissement narcissique sont nombreuses et comportent tous les degrés. Ici, nous interrogerons seulement ces processus qui, par leur intensité, semblent accomplir une forme de meurtre psychique, dépossédant l'enfant d'un espace psychique dans lequel un «je » pourrait advenir. La place de double dans laquelle se trouvent figés certains enfants s'impose comme une des voies privilégiées empruntées par un tel investissement narcissique. Nous verrons, en particulier à travers l'histoire d'Antoine, les différents enjeux psychiques d'un tel fonctionnement. En effet, par-delà cet apparent meurtre psychique, c'est parfois le fantasme d'un enfant meurtrier qui se dévoile à notre écoute, dès lors qu'on ne se laisse pas abuser par les masques divers et variés dont il peut se recouvrir. Ainsi en est-il parfois des figures culturelles de «l'enfant ancêtre » ou de «l'enfant-possédé » qui, dans certains récits, viennent figer une parole plutôt qu'elles ne rendent possible la survenue d'une surprise par laquelle des sens nouveaux pourraient advenir ${ }^{8}$. Mises en avant - comme peuvent l'être certains récits de deuil ou de maladies - elles écrasent le temps et l'espace et maintiennent un fonctionnement adhésif dans lequel l'enfant ne saurait exister autrement que comme figure de double. Ainsi en a-t-il été lors de ma rencontre avec Antoine, 3 ans, et sa

\footnotetext{
${ }^{8}$ Nous n'excluons pas le fait que dans certaines familles le recours à une étiologie mettant en jeu la tradition puisse être le moteur d'une élaboration psychique et d'une transformation, dès lors que ces étiologies trouvent une écoute. Mais tel n'est pas toujours le cas.
} 
mère, originaires d'un pays d'Afrique occidentale. La mère mit d'emblée en avant la relation père-fils, ainsi que l'incapacité de son mari à dire «non » à cet enfant (du fait, entre autres, de leur ressemblance importante), tandis qu'elle éludait son absence de langage, ses difficultés à entrer en relation avec autrui (regard fuyant). Elle me livra, de façon purement descriptive, sans commentaire ni conclusion, toute une série d'interprétations relatives à sa propre culture :

$\checkmark$ Antoine était né après des jumelles, (apparaissant ainsi comme un être doté d'un pouvoir immense ${ }^{9}$ ).

$\checkmark$ Il était né après-terme, (ce qui faisait de lui un sorcier potentiel).

$\checkmark$ La naissance d'Antoine s'était inscrite entre deux morts : celle du grand-père paternel de son père, décédé peu de temps auparavant et celle d'un oncle de la mère, décédé peu après. (Il avait donc doublement le statut d'enfant-ancêtre.)

L'énonciation le plus souvent implicite de telles interprétations culturelles apparaît en partie comme un test concernant la capacité de l'analyste à entendre ce qui n'est pas formulé explicitement et qui appartient à un autre monde. Toutefois, il me semble que lorsqu'une figure culturelle est ainsi mise en avant, sans pouvoir donner lieu à élaboration, l'enjeu se trouve souvent ailleurs. Ne s'agit-il pas alors de déterminer inconsciemment si l'analyste est véritablement autre ou s'il peut être intégré dans un fantasme de non différenciation (ou de faible différenciation) ? De fait, une certaine connaissance des cultures africaines m'a permis, dans ma rencontre avec les parents d'Antoine, de ne pas faire effraction dans la dynamique familiale en apparaissant dans une altérité radicale, bien trop dangereuse et immédiatement persécutrice. Il a donc fallu ce temps au cours duquel allait se déployer le masque protecteur d'une parole culturelle. En formulant, devant cet afflux d'interprétations, qu'Antoine était un enfant «peu ordinaire », j'apparus comme étant à la limite entre nos deux mondes. Les différences s'en trouvèrent partiellement brouillées, rendant possible le transfert.

Ainsi allait apparaître la place de double occupée par Antoine depuis sa venue au monde. En effet, au fur et à mesure que se déroulaient les récits parentaux ${ }^{10}$, je compris qu'il n'existait pas de place pour cet enfant. Plus précisément, la place qui lui était accordée semblait se réduire à celle d'un enfant mort ou d'un enfant non né ${ }^{11}$. En outre, Antoine, par bien des points, semblait exclu de toute triangulation : seul «le deux» pouvait exister. Ainsi, dans

\footnotetext{
${ }^{9}$ Les éléments indiqués entre parenthèses ne furent pas énoncés par la mère mais déduits par moi.

${ }^{10}$ Pendant plusieurs mois, je recevrai, dans le cadre d'une institution, Antoine avec l'un ou l'autre de ses parents, toutes les deux semaines. Ce n'est qu'ensuite que se mit en place une psychothérapie individuelle, au rythme de deux séances hebdomadaires.

${ }^{11} \mathrm{Au}$ moment où sa mère me parlait de ces différentes situations, Antoine mit en scène un bébé marchant dans des chaussures trop grandes ou trop petites...
} 
l'histoire du couple, Antoine apparaissait-il comme conçu par le seul désir maternel. En effet, sa naissance avait fait l'objet d'un conflit dans le couple et la mère avait décidé seule de la poursuite de cette grossesse. Cependant, le père affichait, en réaction, une apparente omnipotence, disant haut et fort : «je suis le père et la mère de mes enfants », niant de la sorte les fonctions maternelles de sa femme. Et lorsqu'il évoquait les premiers mois de ses enfants, il se décrivait comme celui qui avait exercé seul ces fonctions, prenant à son compte l'entière responsabilité de leur développement. Là encore, seul « le deux » était tolérable.

Mais au-delà de l'histoire d'Antoine, se dessina en arrière-plan celle de deux autres enfants : les parents d'Antoine. Aucun d'eux n'avait pu véritablement être reconnu dans son individualité. C'est leur histoire qui allait faire apparaître l'enjeu doublement vital qui se jouait pour eux dans cet investissement narcissique. En effet, il s'agissait à la fois d'écarter la menace que représentait, pour chacun d'eux, toute séparation et d'échapper au meurtre dont l'auteur à venir, dans leur fantasme, n'était autre qu'Antoine. L'histoire de chacun de ces parents témoigne, en effet, de l'ombre portée par eux, une ombre qui n'avait pu leur permettre de se penser tout à fait comme sujet. En fait, j'acquis progressivement l'impression qu'euxmêmes n'avaient d'existence que comme double d'un mort, et se trouvaient ainsi réduits au statut de morts-vivants. En effet, chacun d'eux avait occupé une place impossible, marquée du sceau de la mort. Porteuse d'une maladie congénitale, la mère avait été considérée comme le double d'une tante, morte adolescente de cette même pathologie. Le père, lui, non seulement ressemblait à s'y méprendre à son propre père, mort quand il était enfant, mais portait la mort dans son regard: dans l'enfance, il avait été identifié comme enfant-sorcier. Cela signifiait pour lui que les personnes qu'il voyait, enfant, dans ses rêves ou dans ses pensées mourraient. Vers 7 ans, une cérémonie lui avait «fermé les yeux », faisant disparaître ce pouvoir de mort. Chacun d'eux s'était cependant soumis aux projections parentales dont il faisait l'objet, se construisant sur une faille majeure : leur existence dépendait de la présence d'un double : la mort ou un mort.

Nul hasard certainement dans la formation de ce couple. La figure du double ayant constitué pour chacun d'eux une enveloppe étrange, tout aussi aliénante que protectrice, ils s'étaient trouvés, établissant l'un avec l'autre une entité double. Dans ces conditions, on peut penser que s'était constitué en eux un fantasme d'engendrement du même. N'avaient-ils pas, en premier lieu, donné vie à un autre couple, des jumelles ? Et c'est avec la conception puis la naissance d'Antoine que la situation semble avoir basculé. Le désaccord concernant cette grossesse, puis la venue au monde d'un enfant unique ne pouvait que faire effraction dans un 
système régi par la notion de double, faire signe d'un danger à venir. Antoine semblait ainsi échapper à cette figure et incarner une altérité non seulement insensée mais éminemment dangereuse dans une telle logique familiale. L'investissement narcissique porté par les représentations culturelles semble donc être venu aussitôt dénier à Antoine sa position d'enfant sujet unique, de futur sujet séparé, l'inscrivant, de fait, comme nouvelle figure de mort-vivant.

Le danger représenté par toute individuation s'imposa à travers l'utilisation, si particulière et longtemps énigmatique, du signifiant «beau » par ces deux parents. En effet, j'avais noté la façon inadaptée, infantile qu'ils avaient de l'utiliser, disant à Antoine «pas beau » lorsqu'il refusait quelque chose, adoptant ainsi une expression d'enfant. Le « pas beau » s'imposait en fait devant toute velléité d'individuation. Mais après-coup, je compris que ce mot était chargé de rendre compte d'une non différenciation : était «beau-belle » celui ou celle qui acceptait la confusion. Or, plus Antoine semblait s'individualiser, plus il leur fallait restaurer une unité narcissique, vitale pour eux. En reprenant à leur compte les mots de l'enfant, en adoptant de façon répétée un comportement infantile, n'essayaient-ils pas de se constituer comme le double d'Antoine ? Ces mots que l'enfant utilisait pour se différencier et s'affirmer, ils les lui retournaient en écho, tentative inconsciente de maintenir une indifférenciation. Ils ne devaient d'ailleurs renoncer à cette position qu'en offrant à Antoine un nouveau double : son frère Patrick, né deux ans après lui. C'est au moment où j'écrivais ces lignes qu'Antoine confirma mon hypothèse, me demandant pourquoi son frère ne venait pas en séance avec lui, si je saurais reconnaître son frère déguisé « en Antoine », mettant en scène le prince Antoine et le prince Patrick dans un face à face spéculaire.

\section{Au-delà du meurtre apparent, la figure de l'enfant meurtrier}

L'investissement narcissique défini par Freud ${ }^{12}$ comme l'amour de ce que l'on est/a été/ voudrait être, n'est donc pas seulement venu, dans cette histoire familiale, comme déni d'une disparition future mais comme modalité de survie psychique au présent. C'est avant tout un déni de l'altérité source de danger, qu'a constitué, je crois, cet investissement. La survie de ces parents semblait donc devoir être payée du prix d'un meurtre psychique, par le maintien d'Antoine dans une figure de double. Devenir sujet autonome lui était véritablement interdit. Ainsi Antoine en vint-il à se/me formuler la question suivante : qu'adviendrait-il s'il

\footnotetext{
${ }^{12}$ FREUD, S. 1914. Pour introduire le narcissisme, dans Euvres complètes, t. XII, Paris, PUF, 2005, p. 233.
} 
« grandissait encore plus dans sa tête ?» Ses parents n'en mourraient-ils pas ? Et c'était là ce que mettaient en scène les différents scénarios qu'il offrait à mon écoute, montrant un père menacé de mort par un fils qui grandit. Ainsi, le danger que représentait inconsciemment son autonomie psychique pour ses parents était-il là, reconnu par Antoine.

Peu à peu la mort, omniprésente dans leurs récits, laissait donc apparaître l'image du meurtre comme moteur de leurs relations. Et si ces représentations n'ont pu être le vecteur de l'engagement, par chacun de ces deux parents, d'un travail thérapeutique indépendant de celui de leur enfant, il fut pourtant essentiel qu'elles aient été déposées dans les séances. Ce furent là, pour chacun de ces deux parents, les seules occasions où leur position d'enfant-ancêtre et d'enfant-sorcier fut parlée, et avec elle la souffrance qui, de tout temps, les avait accompagnés. L'histoire du père, en particulier, paraissait hantée par cette idée de meurtre :

- Celui qu'il avait pensé avoir commis sur son père : la mort de son grand-père, après avoir voulu que son épouse avorte, semble avoir eu sur lui un effet d'unheimlich: n'était-il pas encore et toujours cet enfant dont le regard portait la mort ? Sentiment d'unheimlich qui avait constitué le second temps d'un traumatisme, lui révélant alors une question qu'enfant il avait pu écarter : qu'en était-il du rôle de son désir dans la mort de son père? Cette mort qui, survenue brutalement, bien qu'il ait eu des problèmes de santé chroniques, avait été interprétée par la communauté comme l'œuvre d'un sorcier.

- Celui qu'il avait désiré commettre sur ce fils non voulu par lui ; désir dont il restait des traces malgré les différentes formations réactionnelles qu'il avait mises en œuvre. Ainsi en était-il de son fantasme d'être dangereux pour son fils : lorsque son épouse relatait les difficultés plus grandes d'Antoine en sa présence, la réponse qu'il proposait était toujours de s'éloigner : « il vaut mieux que je sois moins à la maison ».

- Celui réalisé symboliquement par sa propre mère à l'égard d'Antoine, lorsqu'elle lui refusait la moindre parcelle d'existence. En effet, pendant longtemps la grand-mère paternelle d'Antoine refusa non seulement de le rencontrer mais même d'entendre parler de lui. Qu'agissait-elle ainsi ? Etait-ce le meurtre désiré autrefois par son fils, le père d'Antoine, auquel elle s'identifiait? Et si ce dernier vivait aussi douloureusement l'attitude de sa mère, n'était-ce pas aussi parce que le déni de l'existence d'Antoine le renvoyait à sa propre histoire d'enfant annulé dans sa subjectivité ?

Les circonstances liées à la naissance d'Antoine, son statut d'être unique, désireux de son individualité, semblaient avoir fait resurgir la figure du sorcier, cet être dangereux qui se nourrit de la mort d'autrui. Si le père d'Antoine avait pu tuer son propre père, comme il le 
croyait inconsciemment, ne risquait-il pas alors de subir lui aussi le même sort ? Ce pas franchi, Antoine devenait ainsi un meurtrier potentiel. Mais nous verrons que ce fantasme obéit à une autre logique que la seule rivalité œdipienne à laquelle il ne saurait être réduit.

On constate en effet dans certaines histoires combien l'enfant apparait d'emblée persécuteur, appréhendé dans le fantasme parental comme détenteur d'une toute-puissance. Tel semble bien être le cas d'Antoine qui a très tôt été associé à une figure d'immortalité, mais aussi de dangerosité extrême. Il en fut de même pour Fatou, une autre petite patiente, dont l'étiologie implicite - la possession par un esprit - allait bien davantage faire apparaitre la terreur vécue par ses parents. Terreur liée à la conviction que Fatou était une meurtrière en puissance. La toute-puissance affichée par elle dans ses différents comportements semblait confirmer ce fantasme : n'allait-elle pas les tuer, l'un ou l'autre ? Et nous le verrons encore avec Ibrahim. Les récits collectifs nous permettent d'ailleurs d'élargir ces hypothèses. Ainsi, qu'il s'agisse de Jésus, de Merlin ${ }^{13}$ ou de Pâris, tous sont menacés de mort parce qu'ils représentent une menace qu'il faut anéantir. Mais peut-on comprendre ces différents récits, tout comme l'histoire d'Antoine, à partir de la seule projection sur l'enfant de la toute-puissance dont ces parents ont autrefois doté leurs propres parents ? En effet, il me paraît surtout essentiel d'entendre dans ce mouvement, qui fait de l'enfant nouvellement né un être tout-puissant, un déni de l'état de « désaide » du nourrisson. Déni qui vise, selon moi, à occulter l'identification à un être impuissant et dépendant dans lequel ils ne peuvent se reconnaître sans se trouver menacés. Ainsi, si l'on reprend les récits mythiques précédemment cités, on peut faire l'hypothèse que c'est bien l'impuissance de l'enfant qui est en jeu : Jésus échappe certes à la mort, mais il n'en va pas de même pour les autres, ceux qu'on a d'ailleurs appelés les « innocents ». Merlin, à la fin de sa vie chante la faim, le froid et l'abandon, prisonnier de la tour de verre dans laquelle Viviane l'a enfermé. Enfant tout-puissant, il retrouve, à la fin du récit, l'état de « désaide » auquel il semblait avoir échappé au début de son existence ${ }^{14}$.

Le péril en jeu pour ces parents semble donc bien être la capacité du nouveau-né à faire émerger la détresse et l'impuissance qu'autrefois ils ont vécues et qui, sans aucun doute, sont restées actives. La toute-puissance affichée de l'enfant, si elle en fait un être dangereux, n'a-telle pas alors pour but inconscient de les préserver de façon rétroactive ? N'est-ce pas là une tentative, par une identification à l'enfant tout-puissant, d'échapper au meurtre ? Car c'est

\footnotetext{
${ }^{13}$ BORON, R. (De), XIII ${ }^{\mathrm{e}}$ siècle. Merlin, Paris, Flammarion, 1994.

${ }^{14}$ MARKALE, J. 1956. Les grands bardes gallois, Paris, Falaize.
} 
bien cet état qui, dans les récits mythiques, préserve l'enfant : ainsi Merlin échappe-t-il au meurtre grâce à son omniscience.

Dans la psychothérapie d'Ibrahim, enfant de deux ans et demi, atteint d'une amyotrophie spinale, ce fantasme se donna à entendre alors que l'enfant venait d'être hospitalisé après des convulsions. Dans un premier temps, Ibrahim était apparu, dans le discours maternel, sous la représentation d'un enfant-ancêtre. Mais cette représentation allait laisser place à celle d'un enfant-serpent. En l'absence de son fils, elle me dit ceci : «quand une femme accouche d'un serpent, elle ne peut en avoir peur même si elle doit l'enrouler autour de sa taille. » Ainsi, Ibrahim devenait-il un être porteur de mort, qui mord ou qui étouffe sa proie. Or, ne disait-elle pas souvent combien le porter lui coupait le souffle? Ce serpent, qu'il lui fallait entourer autour de sa taille, tel un pagne, témoignait donc de ses fantasmes concernant Ibrahim : il lui collait à la peau et risquait de l'étouffer. En outre, si dans son groupe ethnique, on dit de certains nourrissons qu'ils sont des enfants-serpents, on fait des cérémonies pour qu'ils repartent là d'où ils viennent. En aucun cas, on ne garde auprès de soi un tel enfant. Par cette image, cette mère me disait donc la lutte à mort qui s'était engagée entre elle et son fils. Mais qui en sortirait vainqueur? Allait-elle mourir étouffée par la présence de cet enfant-serpent ou était-ce lui qui allait peu à peu être étouffé par les mots maternels ${ }^{15}$ et ses fantasmes autant que par sa maladie ${ }^{16}$ ? Les convulsions se sont inscrites dans cette dynamique relationnelle ${ }^{17}$. Comme le montre cette séquence clinique, le déni du «désaide » de l'enfant, par le clivage qui l'accompagne, entraîne inexorablement ces parents dans une impasse. Alors que le nourrisson leur apparaît revêtu du halo de la toute-puissance et potentiellement dangereux, eux-mêmes retrouvent leur position infantile d'impuissance, offerts au bon vouloir de l'autre. La mort est à venir, un meurtre doit l'arrêter... En effet, si l'on revient à Antoine, on ne peut que souligner cette interaction récurrente entre risque vital et meurtre. Ainsi, Antoine n'a-t-il pas condensé l'image de l'enfant meurtrier et celle de l'enfant mort, tué par son statut d'enfant-ancêtre? On peut également faire l'hypothèse que le fait d'adhérer à la représentation de l'image d'enfant-sorcier-meurtrier avait constitué, pour le père d'Antoine lorsqu'il était enfant, une défense, un renversement lui permettant d'échapper à la représentation d'un enfant impuissant et nié dans sa singularité. La décision de son épouse de

\footnotetext{
${ }^{15}$ Ibrahim n'existait dans la parole maternelle qu'au travers de ses incapacités, de tout ce qu'elle devait faire pour lui.

${ }^{16}$ En effet, dans la forme sévère de l'amyotrophie spinale, les enfants perdent peu à peu toute possibilité de respirer, ce qui les conduit à une mort certaine.

${ }^{17}$ La neurologue qui le suivit alors me fit part de ses doutes quant à une origine strictement neurologique de ces convulsions. Elle me dit aussi son étonnement concernant l'absence de séquelles après des convulsions qui avaient duré plusieurs heures.
} 
lui imposer cette grossesse aurait alors fait resurgir le spectre de l'enfant qu'il avait été, annulé, effacé. Ainsi, en faisant réapparaître l'enfant impuissant qu'avait été son père, Antoine, l'enfant à naître, ne pouvait échapper à cette place : il fallait annuler la menace représentée par lui, reproduire le meurtre ancien pour survivre, échapper à une nouvelle disparition. La figure du double à laquelle il devait être assimilé en serait le moyen.

La réflexion de Winnicott sur «la crainte de l'effondrement» nous permet de comprendre plus précisément les ressorts internes de ce fantasme. Winnicott souligne en effet l'angoisse particulière qui assaille certains sujets. Il s'agit d'une angoisse d'effondrement, d'une mort à venir qui, en réalité, a déjà eu lieu dans le passé ${ }^{18}$. Or, il me semble que ces trois temps se trouvent pareillement engagés dans l'investissement narcissique décrit ici : un meurtre serait à venir, réalisé par l'enfant sur son parent alors que ce meurtre a déjà eu lieu dans l'histoire infantile du parent. S'engagerait alors, à travers la figure du double, l'orchestration d'un meurtre destiné à préserver le parent. Mais le meurtre ayant déjà été accompli, il ne pourrait que se répéter inlassablement au présent. Et c'est bien ce qu'ont donné à entendre les parents d'Antoine : le meurtre dont ils craignaient la réalisation avait déjà eu lieu, autrefois, en un temps où ils n'avaient pu s'y opposer et s'en protéger. Ils l'avaient ainsi subi sans pour autant en prendre conscience : il avait donc fallu tout d'abord le déplacer dans l'avenir pour tenter de s'en préserver puis le perpétrer sur la personne de leur enfant. Il s'agit alors d'une tentative désespérée de survie qui nous montre comment l'enfant se trouve pris au piège d'une histoire dont il peut cependant s'extraire, dès lors que sa parole et celle de ses parents trouvent à se dire dans une relation transférentielle. Ainsi, à travers les récits confiés à mon écoute par les parents d'Antoine, put apparaître leur souffrance d'enfant, depuis toujours méconnue. Cette parole constitua le premier pas vers la réappropriation d'une histoire douloureuse qui les avait tous deux privés d'une existence pleine et entière. De surcroît, elle fut reçue par Antoine qui élabora ainsi les moyens de prendre place, de pouvoir progressivement exister en toute subjectivité.

\section{Conclusion :}

Par la figure de double attribuée à certains enfants, il s'agirait, pour leurs parents, d'écarter le visage menaçant de la différenciation et ce faisant, d'échapper à un meurtrier fantasmé en la

\footnotetext{
${ }^{18}$ Winnicott, D.W. (non daté). La crainte de l'effondrement, dans La crainte de l'effondrement et autres situations cliniques, Paris, Gallimard, 2000, 205-216.
} 
personne de leur enfant. En effet, c'est bien le fantasme d'un enfant meurtrier qui semble, ici et dans d'autres cures, se dessiner. Engageant plusieurs temps, ce fantasme déplacerait dans le futur un meurtre psychique perpétré dans le passé sur ces parents. D'où l'instauration de relations complexes dans lesquelles un duel semble constamment en cours. Qui tuera qui ? semblent se demander ces parents, terrifiés par cette image de leur enfant, qui, en réalité, ne fait qu'endosser le rôle du parent qui, autrefois, les a psychiquement privés de leur individualité. Désormais dépendants d'un double, ils rejouent encore et toujours la même scène avec un enfant qui se trouve, lui, prisonnier d'une image insupportable, désubjectivante.

\section{Résumé}

Dans certaines configurations familiales, la figure de double portée par l'enfant semble accomplir l'équivalent d'un meurtre psychique. Livrée à l'écoute de l'analyste, cette figure dévoile l'investissement narcissique qui la sous-tend. Elle fait également apparaître l'enjeu vital qui se joue pour ces parents. En effet, si cette figure du double leur permet d'évincer toute confrontation à une individuation ressentie comme menace, le meurtre psychique qu'elle semble réaliser a, lui, pour but de les préserver du fantasme d'un enfant meurtrier. En fait, ce fantasme déplace dans le futur le meurtre psychique qu'ils ont eux-mêmes subi dans leur enfance. Afin de se soustraire à la menace toujours à venir d'un tel meurtre, il faudrait donc le perpétrer au présent, sur la personne de l'enfant : la figure du double en serait le moyen.

Mots clefs : investissement narcissique, figure du double, fantasme d'un enfant meurtrier

Narcissistic investment, figure of the "double" and fantasy of a murdered child

Summary : Some children are unconsciously considered as a «double » by their parents. This figure may appear as a psychic murder. Listened by the analyst, this figure of the double reveals a huge narcissistic investment. This is above all for those parents a way of survival. It allows them to dispel any confrontation with alterity, felt as dangerous. Thus realized, this murder aims to preserve them from the fantasy of a murdered child. In fact, this fantasy moves in time the psychic murder, which they endured themselves in youth. In order to avoid this murder threat to come, they would have to perpetrate it nowadays : the figure of the double would be a way to.

Key words : narcissistic investment, figure of the double, fantasy of a murdered child

\section{Bibliographie}

DE BORON, R. XIII ${ }^{\mathrm{e}}$ siècle. Merlin, Paris, Flammarion, 1994. 
FREUD, S. 1914. Pour introduire le narcissisme, dans Euvres complètes, t. XII, Paris, PUF, 2005 214-245.

FREUD, S. 1919. L'inquiétant dans CEuvres complètes, t. XV, Paris, PUF, 1996 147-188.

MANZANO, J., PALACIO ESPASA, F. et ZILKHA, N. 1999. Les scénarios narcissiques de la parentalité, Paris, PUF.

MARKALE, J. 1956. Les grands bardes gallois, Paris, Falaize.

RACAMIER, P.C. 1979. La maternalité psychotique, dans De psychanalyse en psychiatrie, Paris, Payot, 193-242.

RACAMIER, P.C. 1995, L'inceste et l'incestuel, Paris, Les éditions du collège.

WINNICOTT, D.W. (non daté) La crainte de l'effondrement, dans La crainte de l'effondrement et autres situations cliniques, Paris, Gallimard, 2000, 205-216. 